\title{
REFLEXION SPACES AND HOMOGENEOUS SYMMETRIC SPACES
}

\author{
BY OTTMAR LOOS ${ }^{1}$
}

Communicated by R. S. Palais, November 7, 1966

1. A reflexion space is a set $M$ with a multiplication $\mu: M \times M \rightarrow M$, $(x, y) \mapsto x \cdot y$, satisfying the following axioms:

$$
\begin{aligned}
x \cdot x & =x, \\
x \cdot(x \cdot y) & =y, \\
x \cdot(y \cdot z) & =(x \cdot y) \cdot(x \cdot z) .
\end{aligned}
$$

Let be $S(x): y \rightarrow \rightarrow x \cdot y$ the left multiplication with $x$ in $M$. This is an involutive map of $M$ on to itself leaving $x$ fixed, which may be interpreted as the reflexion in the point $x$.

Let $\mathfrak{A}$ be a finite dimensional Jordan algebra and $I$ the set of invertible elements of $\mathfrak{A}$. In general for $x, y \in I$ their product $x y$ is not in $I$, so $I$ does not inherit a multiplicative structure from $\mathfrak{A}$. However, $x \cdot y=2 x\left(x y^{-1}\right)-x^{2} y^{-1}$ is invertible ([1]), and the multiplication $x \cdot y$ makes $I$ a reflexion space. Every group is a reflexion space with the new product $x \cdot y=x y^{-1} x$. Every set is a reflexion space with the trivial product $x \cdot y=y$ for all $x$ and $y$.

A reflexion space $M$ where $M$ is a connected paracompact $C^{\infty}$ manifold and $\mu: M \times M \rightarrow M$ is differentiable is called a differentiable reflexion space. The following construction gives examples. Let $G$ be a connected Lie group, $\sigma$ an involutive automorphism of $G$ and $H$ a subgroup of $G$ lying between the group of all fixed points of $\sigma$ and its identity component. Then $G / H$ is a homogeneous symmetric space and $G(G / H, H)$ is a principal fibre bundle with base space $G / H$ and structure group $H$. Let $H$ operate on a connected manifold $F$ on the left and let be $G \times{ }_{H} F$ the bundle associated with $G(G / H, H)$ with typical fibre $F$ (cf [2]). We denote the equivalence class of $(g, x)$ $\in G \times F$ in $G \times{ }_{H} F$ by $g \otimes x$. In case $F$ is a point, we have $G \times{ }_{H} F=G / H$.

Proposition 1. $G \times_{H} F$ is a differentiable reflexion space with the multiplication

$$
(f \otimes x) \cdot(g \otimes y)=\left(f\left(f^{\sigma}\right)^{-1} g^{\sigma}\right) \otimes y .
$$

1 This work is a generalization of part of the author's doctoral dissertation at the University of Munich under Professor M. Koecher. 
Our main result is that all differentiable reflexion spaces are of the type described above (Theorem 2). Proofs will be given in a forthcoming article.

2. A differentiable map $\phi: M \rightarrow N$ of differentiable reflexion spaces $M, N$ is called a homomorphism, if $\phi(x \cdot y)=\phi(x) \cdot \phi(y)$. Isomorphisms and automorphisms are defined as usual. Let Aut $M$ be the group of (differentiable) automorphisms of $M$ and $G$ the group generated by the maps $S(x) \circ S(y), x, y \in M$. We denote the image of a tangent vector $X$ of $M$ under the differential of the map $x i \rightarrow p \cdot x$ by $p \cdot X$; similarly $X \cdot p$ is defined. A vector $X \in T_{p}(M)$ is called vertical (hor $i$ zontal), if $p \cdot X=X$ (resp. $p \cdot X=-X)$. The set $T^{+}(M)$ of vertical vectors (resp. $T^{-}(M)$ of horizontal vectors) form subbundles of the tangent bundle $T(M)$ and we have $T(M)=T^{+}(M) \oplus T^{-}(M)$. Let $J$ be the projection on $T^{-}(M)$ and

$$
S(X, Y)=J[J X, Y]+J[X, J Y]-[J X, J Y]-J[X, Y]
$$

the Nijenhuis torsion tensor of $J$.

Proposition 2. $T^{+}(M)$ is an involutive subbundle of $T(M) . T^{-}(M)$ is involutive if and only if $S=0$.

Let $F_{p}$ be the maximal connected integral manifold of $T^{+}(M)$ through $p$, called the fibre through $p$. It can be characterized as the connected component of $p$ of the set of fixed points of $S(p)$. The set $M_{0}$ of all fibres becomes a reflexion space with the multiplication $F_{p} \cdot F_{q}=F_{p \cdot q}$.

3. Let $M$ be a differentiable reflexion space, $e$ a fixed point of $M$ and $F=F_{e}$ the fibre through $e$. For $X \in T_{e}(M)$ let $L(X)$ be the vector field given by

$$
L(X)_{p}=\frac{1}{2} X \cdot(e \cdot p)
$$

and $g$ the Lie algebra generated by the vector fields $L(X)$. Let $G$ denote the group generated by the transformations $S(x) \circ S(y)$, $x, y \in M$.

THEOREM 1. G has a unique structure of a connected Lie transformation group of $M$, so that its Lie algebra is isomorphic with g. It permutes the fibres transitively. The map $\sigma: g \mid \rightarrow S(e) \circ g \circ S(e)$ is an involutive automorphism of $G$ and the subgroup $H$ of $G$ which leaves $F$ invariant lies between the group of fixed points of $\sigma$ and its identity component.

We now state our main result. 
TheOREM 2. $M$ is isomorphic as a reflexion space with $G \times_{H} F$ under the map $g \otimes x \rightarrow \rightarrow g(x) \quad(g \in G, x \in F)$. The set of fibres $M_{0}$ is isomorphic with $G / H$ under $g H \longmapsto F_{g(e)}$, and the diagram

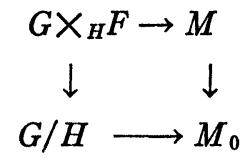

is commutative. Moreover, $M_{0}$ depends functorially on $M$.

4. We keep the notation of the preceding sections. A differentiable reflexion space $M$ is called torsion free, if $S=0$. The maximal connected integral manifolds of $T^{-}(M)$ (Proposition 2) are called the leaves of $M$.

Proposition 3. $S=0$ If and only if the identity component $H^{0}$ of $H$ operates trivially on $F$.

By a result of Koh [3] $H / H^{0}$ is finite. Let be $\Gamma$ the finite group of diffeomorphisms of $F$ induced by $H / H^{0}$. A point of $F$ is called regular if its isotropy subgroup in $\Gamma$ consists of the identity alone, singular otherwise. For the notion of a regular leaf see [4].

TheOREM 3. Let $M$ be torsion free. The leaves are closed submanifolds and coincide with the orbits of $G$. A leaf is regular if and only if it intersects $F$ in a regular point. The restriction of the canonical projection $M \rightarrow M_{0}$ to a leaf $B$ is a finite covering map $B \rightarrow M_{0}$, which is regular with group $\Gamma$ if $B$ is regular. If $M_{0}$ is simply connected, then $M$ is isomorphic with $M_{0} \times F$, where $F$ has the trivial multiplication (see \$1).

A vector field $X$ on $M$ is called a derivation, if

$$
X_{p \cdot q}=X_{p} \cdot q+p \cdot X_{q}
$$

for $p, q \in M$. The set of derivations is a Lie algebra Der $M$. In general the automorphism group Aut $M$ is too big to be a Lie group. However we have

Theorem 4. Let $G$ be transitive on $M$. Then Aut $M$ has a unique structure of a Lie transformation group of $M$ so that its Lie algebra is isomorphic with Der $M$.

\section{REFERENCES}

1. H. Braun and M. Koecher, Jordan-Algebren, Springer, Berlin, 1966.

2. S. Kobayashi and K. Nomizu, Foundatıons of differential geometry. I, Interscience, New York, 1963. 
3. S. S. Koh, On affine symmetric spaces, Trans. Amer. Math. Soc. 119 (1965), 291309.

4. R. S. Palais, $A$ global formulation of the Lie theory of transformation groups, Mem. Amer. Math. Soc. No. 22, 1957.

Mathematisches INSTITUT DER UNIVERSität MÜNCHEN AND UNIVERSITY OF MINNESOTA 